\title{
Estimating Method for Lithium Ion Battery State of Charge based on Twin Support Vector Regression
}

\author{
Yongqin Zhou ${ }^{1, a}$, Yongchao Wang ${ }^{1}$, Guijun Shi ${ }^{2, b}$ and Meilan Zhou ${ }^{1}$ \\ ${ }^{1}$ Harbin University of Science and Technology, Harbin, China \\ ${ }^{2}$ State Grid of YingKou Power Supply Compony,Harbin, China \\ a38973078@qq.com, b2693718376@qq.com
}

\begin{abstract}
SOC is an important parameter of battery, which occupies an important position in BMS. This paper presents a modified method-Twin Support Vector Regression,based on SVR.To pick up current,voltage,ampere-hour, overfitting SOC of lithium ion battery (LIB), and making comparasions with traditional SVR. The results of simulation and experient prove that T-SVR has a higher modeling accuracy, a higher prediction accuracy, and it has a faster computing speed than SVR. TSVR is fit for prediction of SOC of LIB.
\end{abstract}

Keywords: SOC,SVR,Twin Support Regression,lithium ion battery

\section{Introduction}

Lithium ion battery has become one kind of high-tech products in recent years .It has characteristics of high specific energy, high voltage, long cycle life and non memory effect, and no environment pollotion, so it is called" Green Battery". It shows his competitive power and prospect in the day when the environment is polluted seriously. Its dynamic performance can satisfy working condition of Hybrid Electric Vehicle (HEV).

The estimating of HEV battery SOC plays an important role in Battery Management System (BMS). It has an important influence in predicting the remaning journey and avioding battery to overcharg and overdischarg. So, to predict battery SOC well is a key to improve vehicle performance. It concerns auto-industry of our country.

Some existing methods of the estimating of SOC: (1) Open circuit voltage method(OCV) and Ampere-hour integral method (Ah) [1]:OCV is an easy method, but it needs to stewing battery, so it can't satisfy on-line testing. Ah can achieve a good effect in a short time, but it can't get initial value, and as time increases, the error will increase, too. (2) Impedance method [2]: To inflict AC signal for battery and measure impedance spectroscopy in different SOC of LIB. To use these impedance spectroscopy to get corresponding SOC. But this metohd will be influenced by temperature, and it is hard to test when the HEV is running. (3) Equivalent circuit model method [3]: By mainly analysing characteristic of constant current discharge of battery, then we can calculate SOC. But this method relies on veracity of model excessively, meanwhile parameters of model is setted in static state, so it can't reflect the dynamic characteristic of HEV. (4) Nerual Network method [4] (NN): By establishing some parameters of battery with SOC, it can obtain higher estimating accuracy, however, this method relies on men's prior knowledge and the stand or fall of quantity of sample. Meanwhile, it falls into local minimum easily. 
In 1995, Vapnik and his research put forward a new Machine Learning method, called Support Vector Machine(SVM) [5], based on Stasistical Learning Theory ${ }^{[6]}$ SVM is built on the foundation of VC Dimension and Structural Risk Minimization [7], which can solve the problems of nonlinnearity, high dimension, small sample. At the same time, it can get a good generalization ability. SVM extends into regression problem, which emerge a branch algorithm, called Support Vector Regression [8]. Twin Support Vector Regression [9] (TSVR) is an extension method of SVR.By constructing a pair of hyperplanes which separately deterrmine a $\varepsilon$ insensitive upper bound and lower bound. So, TSVR just needs optimize a pair of smaller quadratic programming problem (QPP). So, arithmetic speed of TSVR is faster, generalization ability is better, and the error is smaller.this paper will apply TSVR in estimating of SOC of LIB, modeling and simulinking in the MatLab.

\section{Introduction of SVR and TSVR}

Setting a training sample set $\mathrm{S}$;

$$
S=\left\{\left(x_{1}, y_{1}\right), \ldots,\left(x_{l}, y_{l}\right)\right\} \subset R^{n} \times R
$$

According to given sample set $\mathrm{S}$, What is called regression problem is to find a $f(x)$ which is on $\mathrm{R}^{\mathrm{n}}$ and use $y=f(x)$ deduce given $x$ corresponding $y$

According to Mercer theory, it can use kernel function $K(x, y)$ to define inner product where is on $\mathrm{H}$, which is $K(x, y)=\langle\phi(x), \phi(y)\rangle$.

To SVR, we expect to achieve regression function:

$$
f(x)=\langle\phi(x), \omega\rangle+b
$$

To aviod the loss of sample points which lie out of blank, it defines a $\varepsilon$ insensitive loss function to soften the blank. So minimized restricted problem of SVR is:

$$
\begin{gathered}
\min \frac{1}{2}\|\omega\|^{2}+C \sum_{i=1}^{l}\left(\xi_{i}+\xi_{i}^{*}\right) \\
\text { s.t. } f\left(x_{i}\right)-y_{i} \leq \xi_{i}^{*}+\varepsilon \\
y_{i}-f\left(x_{i}\right) \leq \xi_{i}+\varepsilon \\
\xi_{i}^{*}, \xi_{i} \geq 0
\end{gathered}
$$

$\mathrm{C}$ is punishment parameter, and $\mathrm{C}>0 . \xi_{i}^{*}, \xi_{i}$ is slack variable which can reflect if sample points land in $\varepsilon$ insensitive region.

According to solve Dual Problem of above-mentioned problems, we can achieve solution. Its Dual Problem is:

$$
\max -\frac{1}{2} \sum_{i, j=1}^{l}\left(\alpha_{i}-\alpha_{i}^{*}\right)\left(\alpha_{j}-\alpha_{j}^{*}\right) K\left(x_{i}, x_{j}\right)-\sum_{i=1}^{l}\left(\alpha_{i}+\alpha_{i}^{*}\right) \varepsilon+\sum_{i=1}^{l}\left(\alpha_{\mathrm{i}}-\alpha_{i}^{*}\right) y_{i}
$$




$$
\begin{aligned}
& \text { s.t. } \sum_{i=1}^{l}\left(\alpha_{i}-\alpha_{i}^{*}\right)=0 \\
& 0 \leq \alpha_{i}, \alpha_{j} \leq C, \mathrm{i}=1, \ldots 1
\end{aligned}
$$

$\alpha_{i}, \quad \alpha_{i}^{*}$ is lagrangian multiplier.

Find out this Dual Problem, then we can achieve the last regression function (2) .

On the basis of training set (1),TSVR can make two hyperplanes which can determin the last hyperplane. We can suppose that there is a row vector $A_{i}, \mathrm{i}=1,2,3 \ldots . .1$ and it means that they are 1 samples in $\mathrm{R}^{\mathrm{n}}$, among them ,the ith sample is $A_{i}=\left(A_{i 1}, \ldots \ldots, A_{i n}\right)$. $A=\left(A_{1}, \ldots ., A_{l}\right), Y=\left(y_{1}, \ldots . y_{l}\right)^{T}, y_{i} \in R$ means output value of sample. Now we give training sample $(A, Y)$, then training $\varepsilon_{1}-$ insensitive lower function: $f_{1}(x)=K(A, x) \omega_{1}+b$ and $\varepsilon_{2}$-insensitive upper function: $f_{2}(x)=K(A, x) \omega_{2}+b_{2}$ of data ,here and now we can determine the final regression function. The average value of these two funtions' sum is the final regression funtion. Function $f_{1}(x)$ and $f_{2}(x)$ is determined by these two quadratic programmings.

$$
\begin{aligned}
& \quad \min \frac{1}{2}\left\|Y-e \varepsilon_{1}-\left(K\left(A, A^{T}\right) \omega_{1}+e b_{1}\right)\right\|^{2}+C_{1} e^{T} \zeta, \\
& \text { s.t. } \quad Y-\left(K\left(A, A^{T}\right) \omega_{1}+e b_{1}\right) \geq e \varepsilon_{1}-\zeta, \zeta \geq 0 \\
& \quad \min \frac{1}{2}\left\|Y+e \varepsilon_{2}-\left(K\left(A, A^{T}\right) \omega_{2}+e b_{2}\right)\right\|^{2}+C_{2} e^{T} \eta, \\
& \text { s.t. } \quad\left(K\left(A, A^{T}\right) \omega_{2}+e b_{2}\right)-Y \geq e \varepsilon_{2}-\eta, \eta \geq 0 .
\end{aligned}
$$

$C_{1}, C_{2}>0, \varepsilon_{1}, \varepsilon_{2} \geq 0$ are given as certain parameters, $\zeta, \eta$ are slack variable,e is the unit column vector, introducing lagrangian multiplier vector $\alpha, \gamma$,combining with KKT condition, we can achieve quadratic programming problem of dual problem of equation(5).

$$
\begin{gathered}
\max -\frac{1}{2} \alpha^{T} H\left(H^{T} H\right)^{-1} H^{T} \alpha-h^{T} H\left(H^{T} H\right)^{-1} H^{T} \alpha-h^{T} \alpha \\
\text { s.t. } 0 \leq \alpha \leq C_{1} e .
\end{gathered}
$$

and $H=\left\lfloor K\left(A, A^{T}\right), e\right\rfloor, \quad h=Y-e \varepsilon_{1}$

optimizing this dual problem,we can achieve,

$$
\left[\omega_{1}^{T}, b_{1}\right]^{T}=\left(H^{T} H\right)^{-1} H^{T}(h-\alpha),
$$


Therefore, according to the same conditon quadratic programming problem of dual problem of equation (6) is ,

$$
\begin{gathered}
\max -\frac{1}{2} \gamma^{T} H\left(H^{T} H\right)^{-1} H^{T} \gamma-g^{T} H\left(H^{T} H\right)^{-1} H^{T} \alpha-g^{T} \alpha \\
\text { s.t. } 0 \leq \gamma \leq C_{2} e ., \quad g=Y+e \varepsilon_{2}
\end{gathered}
$$

optimizing this problem,

$$
\left[\omega_{2}, b_{2}\right]^{T}=\left(H^{T} H\right)^{-1} H^{T}(g+\gamma)
$$

so the regression function of TSVR is,

$$
f(x)=\frac{1}{2} K(A, x)\left(\omega_{1}+\omega_{2}\right)^{T}+\frac{1}{2}\left(b_{1}+b_{2}\right) .
$$

$\omega_{1}, \omega_{2}$ is weight coefficient, which is determined by lagrangian multiplier vector $\alpha, \gamma$, and $\alpha, \gamma, b_{1}, b_{2}$ are known quantities.A is input value.

\section{The Battery Model Framework and Parameter Selection}

When battery is in the charge and discharge progress, BMS can get current(i), voltage(v), and net $\mathrm{Ah}$ of battery(Ah). Net $\mathrm{Ah}$, which is a representation of basic parameters of battery charge and dis charge, is got by Ah integral method. Because of the reason of current measurement error, it leads to measure net Ah unaccurately usually, and with the net Ah error accumulating, the error will expand. So I decide to use i, v, and Ah of battrery as input value .Meanwhile SOC is output value.

$$
A=\left[\begin{array}{ccc}
i_{1} & u_{1} & A h_{1} \\
i_{2} & u_{2} & A h_{2} \\
\cdot & \cdot & \cdot \\
\cdot & \cdot & \cdot \\
i_{n} & u_{n} & A h_{n}
\end{array}\right] \quad Y=\left[\begin{array}{l}
S O C_{1} \\
S O C_{2} \\
\cdot \\
\cdot \\
S O C_{3}
\end{array}\right]
$$

Then we can establish the function relationship of input/output: $Y=f(A)$.

Before establishing model,we must select kernel function.This paper selects RBF kernel function, $K\left(x_{i}, x_{j}\right)=e^{-\gamma\left\|x_{j}-x_{i}\right\|^{2}}$.

The parameters of TSVR include penalty parameter $\left(C_{1}, C_{2}\right)$, loss parameter $\left(\varepsilon_{1}, \varepsilon_{2}\right)$, and ker nel function $\gamma$.Usually,we stipulate $C_{1}=C_{2}, \varepsilon_{1}=\varepsilon_{2}$, then according to cross validation 
to optimize.First, we determine a parameter(usually, $\gamma=2.0$ ), and use cross validation to determine extra two parameters,at last,we can get $C_{1}=C_{2}=200, \varepsilon_{1}=\varepsilon_{2}=0.01$.

The framework maps of estimating method for LIB SOC based on TSVR will be as below:

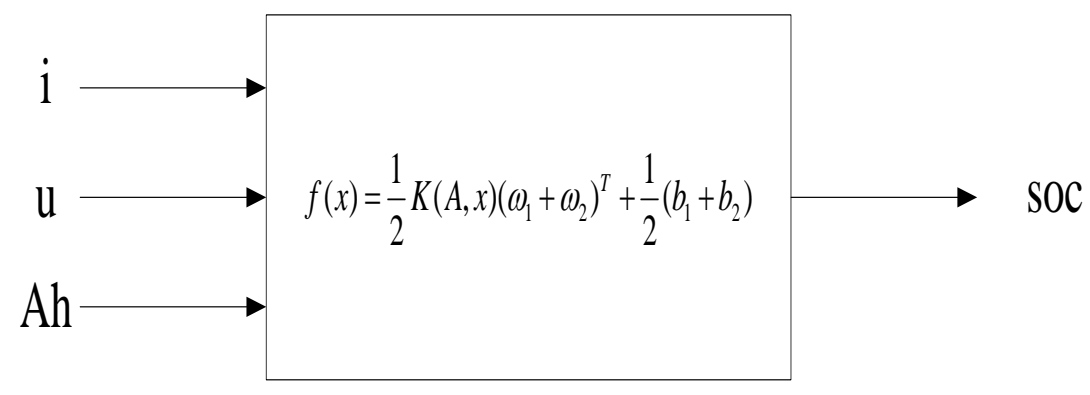

Figure 1. Framework maps

\section{Modeling}

In the experiment, we select lithium iron phosphate, which type is 32650 ,diametre is $32 \mathrm{~mm}$, length is $650 \mathrm{~mm}$, rated capacity is $5 \mathrm{Ah}$, rated voltage is $3.2 \mathrm{~V}$, and charge-discharg rate is $2 \mathrm{C}$. Start the experiment, and record the data. In the $1 \mathrm{C}$ charge data of battery, we select 20 sample points homogeneously. Meanwhile in order to prove superiority of TSVR, we use SVR to establish model in the same data. Absolute error of two methods will be compared, too.

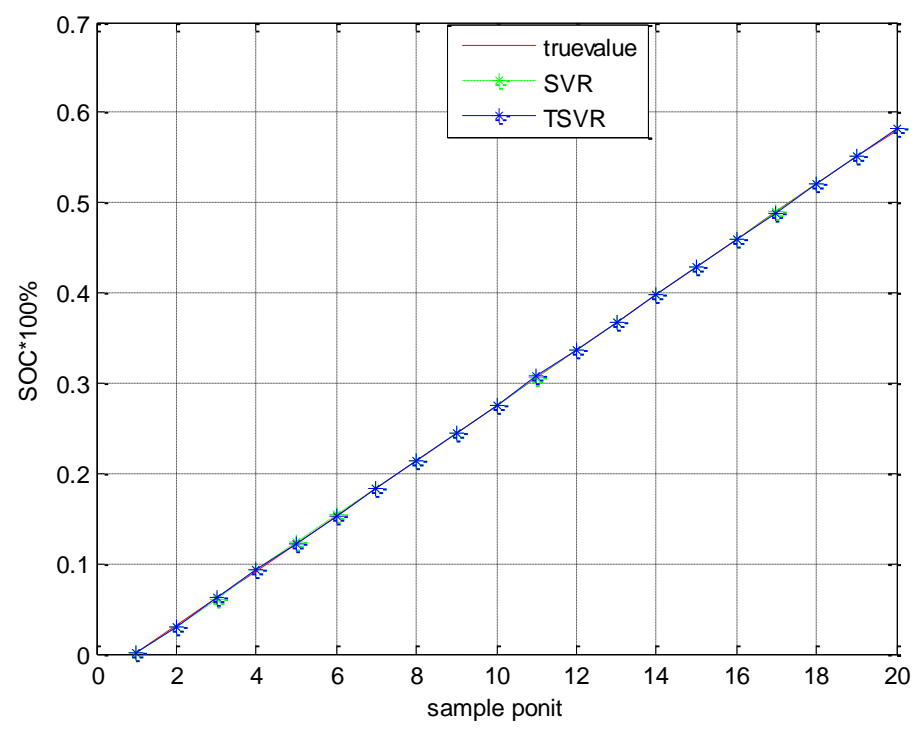

Figuer 2. $1 \mathrm{C}$ modeling 


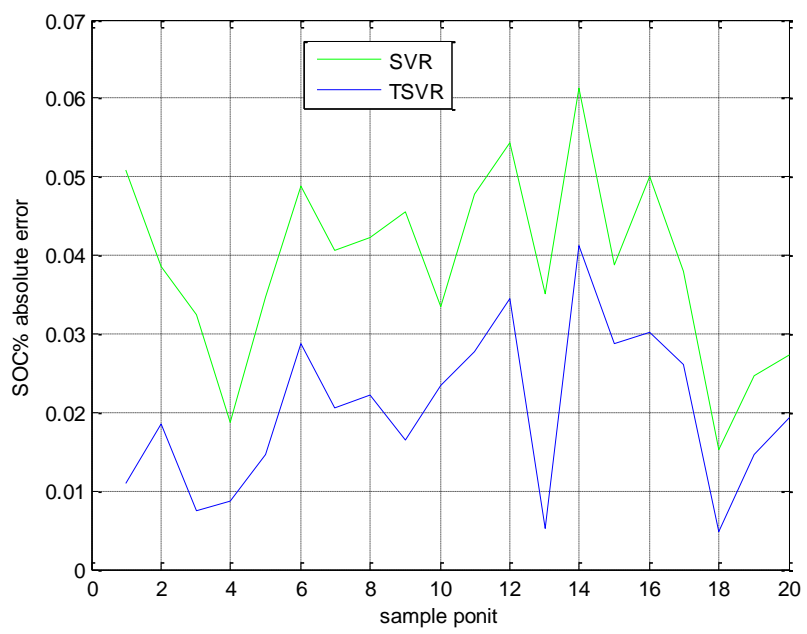

Figure 3. 1C absolute error

From the 2 pictures, we can find that TSVR modeling presicion is very high, and its maximum modeling error is $0.04213 \%$ and its homogeneous error is $0.01217 \%$. The absolute error of TSVR is less than SVR, too.

\section{Prediction}

Now, I have used $0.2 \mathrm{C}, 0.4 \mathrm{C}, 0.6 \mathrm{C}, 1 \mathrm{C} .1 .4 \mathrm{C}$ rate to establish model, meanwhile I use $0.3 \mathrm{C}, 0.5 \mathrm{C}, 0.8 \mathrm{C}, 1.2 \mathrm{C}, 2 \mathrm{C}$ rate to predict $\mathrm{SOC}$ of the battery and plot chart of prediction, and compare with SVR. The experiment will use the method of charge-standing-charge, First, using $0.3 \mathrm{C}$ to charge, and stand. Then, using $0.5 \mathrm{C}$ to charge, and stand. Using $0.8 \mathrm{C}$ to charge, and stand. Gradually to $2 \mathrm{C}$. In each rate, wo select 20 sample points homogeneously. Predicted curve should be something like that in Figure 4.

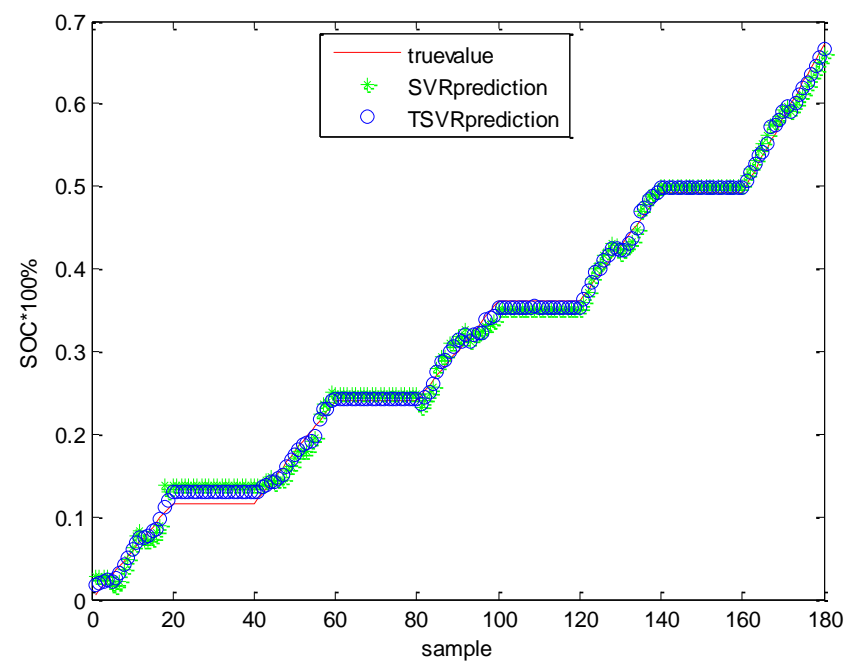

Figure 4. prdicted curve 
From the Figure 4, the absolute error curve, we can see that predicted error of TSVR is less than SVR, and fluctuation range of TSVR is smaller than SVR. Totally, it can show TSVR is better than SVR.

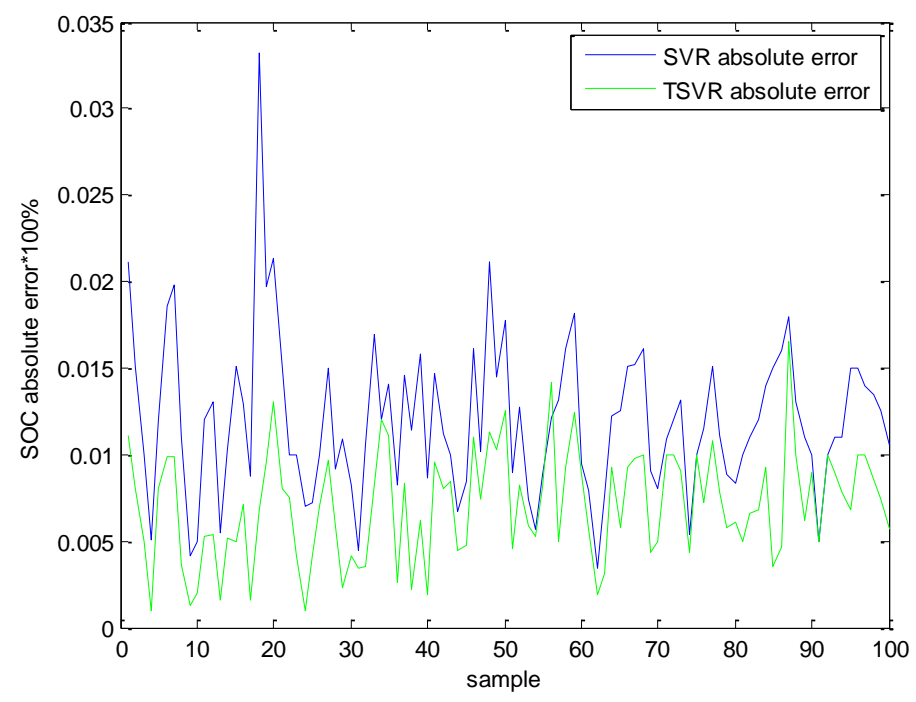

Figuer 5. Predicted curve of absolute error

In Table 1, we can see many comparisons in error part between TSVR and SVR. In error parts, we know, TSVR is more superiorer than SVR.

Table 1. Comparisons in error part between TSVR and SVR

\begin{tabular}{|l|l|l|}
\hline & \multicolumn{1}{|c|}{ TSVR } & \multicolumn{1}{|c|}{ SVR } \\
\hline The maximum modeling error SOC \% & 0.04213 & 0.06341 \\
\hline The maximum predicted error SOC \% & 1.68973 & 3.33715 \\
\hline The average modeling errorSOC \% & 0.01207 & 0.03137 \\
\hline The average predicted errorSOC \% & 1.17692 & 2.11671 \\
\hline
\end{tabular}

\section{Conclusion}

This paper introduces a new method for predicting SOC of battery,TSVR, which is based on SVR, and applies this method in preidicting SOC of LIB BMS of HEV.In math method,since TSVR converts optimizing a large hyperplane into optimizing two small hyperplanes,it shortens operation time.According to analyze data of the experiment,comparing TSVR with $\mathrm{SVR}$, the former has higher modeling precision and stronger generalization ability.Providing a basis for predicting SOC of battery, and in a way ,it has reference value.

\section{Acknowledgements}

This work is supported by Heilongjiang Natural Science Foundation(E201302). 


\section{References}

[1] J. H. Aylor, A. Thieme and B. W. Johnson, "A battery state of charge indicator for electric wheelchairs", IEEE, vol. 39, (1992), pp. 398-409.

[2] A. J. Ruddell, A. G. Dutton and H. Wenzl, "Analysis of battery current microcycles in autonomouss renewable energy systems", Journal of Power Source, vol. 112, (2002), pp. 531-546.

[3] M. S. Ziyad, A. C. Margaret and A. L. William, "A mathematical model for lead-acid batteries", IEEE Transactions on Energy Conversion, vol. 7, no. 1, (1992), pp. 63-67.

[4] W. X. Shen, K. T. Chau and C. C. Chan, "Neural network based residual capacity indicator for nickel-metal hydride batteries in electric vehicles", IEEE Trans on Vehicular Technology, vol. 54, no. 5, (2005), pp. 1705-1712.

[5] D. Naiyang and T. Yingjie, "New methods in data mining support vector machine", Beijing: Science Press, (2004), pp. 224-226.

[6] B. Scholkopf and A. Smola, "Learning with kernels", Cambridge, MA: MIT Press, (2002).

[7] V. N. Vapnik, "Statistical learning theory", New York: Wiley, (1998).

[8] P. Xinjun, "TSVR: an efficient twin support vector machine for regression", Neural Networks, vol. 23, no. 3, (2010), pp. 365-372.

[9] V. N. Vapnik, “The natural statistical learning theory”, New York: Springer, (1995). 